\title{
Process of Parentification and Construction of Ego Identity in the Cameroonian Adolescents
}

\author{
${ }^{1}$ Ndje Ndje Mireille, ${ }^{2}$ Ndzerem Shela Shiyghan, ${ }^{3}$ Tsala Tsala Jacques Philippe \\ ${ }^{1} \mathrm{Ph} / \mathrm{D}$ in Clinical and Pathological Psychology, Senior Lecturer, University of Yaoundé I, Department of \\ Psychology. ${ }^{2}$ Master in Clinical and Pathological Psychology, University of Yaounde I, Department of \\ Psychology. ${ }^{3}$ Full Professor, University of Yaounde I, Head of Department of Psychology.
}

\begin{abstract}
Culture and idiosyncratic family configuration play a significant role in neglect, such as parentification. Some family systems may engender an inappropriate overlap in subsystems, with member participating in roles that are traditionally reserved for other members. We have the case of adolescents in parental roles. The adolescent is brought to make personal choices that will have long term repercussions on varied domains of his life. To this effect, the society should give to him the possibility of a psycho-social moratorium which is a necessary period for the construction of a true identity. So how the construction of self-identity would be effectuated in the case of parentified adolescents. We carried out a semi-structured interview on five adolescents in Yaounde, the capital city of Cameroon who are in a situation of parentification. For the analysis, we used the content as well as the thematic analysis of the interviews. The findings show that the parentified adolescent have had no time to live the stage of adolescence. They are early parents because of the responsibilities they carry at an age or time they are not ready or prepared for. What is particular and special about them is the extent to which they are able to construct a positive self and give positive self-view of life. So, the process of parentificatin will have positive repercussions on the construction of ego identity of the adolescent. The findings of this study has shown the importance of contextual study as a good number of literature on parentification have shown the destructive and maladaptive impact of parentification on the development of adolescents.
\end{abstract}

Key words: Adolescence, ego identity, family, parentification, responsibility. 